\title{
APPLICATION OF A FEM-BEM COUPLING METHOD IN STEADY-STATE HEAT TRANSFER PROBLEM
}

\author{
QI HE ${ }^{1}$, YANPENG GONG ${ }^{1,2} \&$ FEI QIN ${ }^{1,2}$ \\ ${ }^{1}$ Institute of Electronics Packaging Technology and Reliability, Faculty of Materials and Manufacturing, \\ Beijing University of Technology, China \\ ${ }^{2}$ Beijing Key Laboratory of Advanced Manufacturing Technology, China
}

\begin{abstract}
In this paper, an FEM-BEM coupling method is presented to study the steady-state heat transfer problem. In the analysis of some complex structures, the FEM-BEM coupling method has many advantages. These advantages are: (i) The degrees of freedom can be highly reduced using the boundary elements; (ii) The improved accuracy of solution over classical FEM; (iii) The use of powerful pre-/postprocessing module of the finite element software (ABAQUS); (iv) The improved efficiency of solving multiscale structures. In this work, the user-defined element (UEL) subroutine in ABAQUS is used to realize the coupling of ABAQUS and BEM. The model is divided into several parts and different methods will be used over different part. In the implementation of the coupling scheme, BEM part can be assembled into the ABAQUS as a super-element. And the coupling stiffness matrix which is consisted of the true stiffness matrix of BEM and the stiffness matrix of FEM can be obtained. Then the resulted system equations can be solved by the solver of ABAQUS. Results obtained by this coupling method have an excellent agreement compared with the analytical or reference solutions.
\end{abstract}

Keywords: FEM-BEM coupling, ABAQUS, UEL, steady-state heat transfer.

\section{INTRODUCTION}

With the development of science and technology, semiconductor structures are widely used in many areas. Since semiconductor structures are sensitive to the change of temperature, thermal analysis of these structures has been widely studied [1]. In recent years, numerical analysis of semiconductor structures is becoming a hot topic caused by the characteristic of its simplicity, effectiveness and lower cost. Furthermore, the results obtained by numerical techniques can provide some important guidances for the design of semiconductor structures. However, for the numerical analysis of semiconductor structures, large number of multiscale structures usually exist in a numerical model, which results in a big challenge for engineers and researchers [2].

Up to now, many numerical schemes have been used to study multiscale structures including finite element method (FEM), boundary element method (BEM), etc. In the thermal analysis of the electronic devices with multiscale structures by finite element method (FEM), large number of elements is needed to improve the accuracy of the results, which increases the computing time sharply [3]. To solve some practical engineering problems by FEM, many commercial tools are developed such as ABAQUS, ANSYS etc. All these tools can provide powerful pre-/post-processor, which has attracted many engineers. BEM is also a useful numerical technique for the solution of partial differential equations, offering an alternative to the FEM for a range of engineering simulations [4]. The main advantages of the BEM derive from the fact that its approximations (and mesh) only occur on the boundary, the dimension of the numerical model therefore being one less than that of the physical problem being modelled, and from the high accuracy of its solutions on comparatively coarse meshes [5][9]. These advantages suggest the BEM can be effectively applied to the analysis of multiscale structures. Actually, the coupling of FEM and BEM is also a powerful method for the thermal analysis of the semiconductor structures. In 1977, Zienkiewicz et al. proposed the coupling of 
FEM and BEM to benefit from the combination of two methods [10]. Then, different coupling methods are presented to solve all kinds of problems. In [11], Estorff and Firuziaan apply the coupling BEM/FEM for nonlinear soil/structure interaction. Elleithy et al. proposed the iterative coupling in the elastic statics and elastic plasticity problems [12], [13]. Godinho and Soares also used the coupling method to solve the problem in soil-structure elastic dynamic interaction [14]. In [15], Liu and Dong presented an automatic implementation procedure for the coupling of the ABAQUS with a self-written linear elastic BE code for dynamic elastoplastic problems. There are also many BEM-FEM coupling procedures, applied in nonlinear, dynamic, complex interface and fluid/structure problems, that have been reported in recent years [16]- [19].

In this paper, based on the commercial finite element analysis (FEA) software (ABAQUS), we propose a coupling method of FEM-BEM and apply the scheme to a heat transfer problem. In the implementation of the coupling, the BE region is defined as a large finite element and its stiffness is computed and assembled into the global stiffness matrix of FEM. The stiffness of the model from BE region is computed by the BEM code. To realize the integration of BEM and FEM, the BEM code is put into user-defined element (UEL) subroutine provided by ABAQUS. A classical heat transfer problem is presented to demonstrate the correctness of this method.

\section{THEORETICAL ANALYSIS}

Many physical problems, such as electromagnetic problems, heat conduction, seepage and acoustic problems, can be described by classical Laplace equations or Poisson equations. For a $2 \mathrm{D}$ problem in $\Omega \in \mathbb{R}^{2}$ with closed boundary $\Gamma$, the Laplace equations can be expressed as follows:

$$
\nabla^{2} u(\mathbf{x})=\frac{\partial^{2} u}{\partial x_{1}^{2}}+\frac{\partial^{2} u}{\partial x_{2}^{2}}=0
$$

where $\nabla^{2}$ is the Laplace operator. $u(x)$ is the potential function or temperature at point $\mathbf{x}\left(x_{1}, x_{2}\right) \in \Omega$.

Eqn. (1) can be solved subject to a set of boundary conditions taken from the following:

$$
\begin{gathered}
u(\mathbf{x})=\bar{u}(\mathbf{x}) \quad \text { on } \quad \mathbf{x} \in \Gamma_{1}, \\
t(\mathbf{x})=k \frac{\partial u}{\partial \mathbf{n}}=\bar{t}(\mathbf{x}) \quad \text { on } \quad \mathbf{x} \in \Gamma_{2},
\end{gathered}
$$

where $k$ is the thermal conductivity; $t(x)$ is the heat flux. $\mathbf{n}$ is the outward pointing normal. The quantities $\bar{u}$ and $\bar{t}$ are known temperature and heat flux, respectively. $\Gamma=\Gamma_{1} \cup \Gamma_{2}$ and $\Gamma_{1} \cap \Gamma_{2}=\phi$.

\subsection{FE formulations for the steady-state heat transfer problem}

For the finite element solution of the heat transfer problem, we can obtain the following functional expressions based the conclusion in [20]:

$$
\Pi(u)=\int_{\Omega}\left[\frac{1}{2} k\left(\frac{\partial u}{\partial x_{1}}\right)^{2}+\frac{1}{2} k\left(\frac{\partial u}{\partial x_{2}}\right)^{2}\right] d \Omega-\int_{\Gamma_{2}} t u d \Gamma .
$$

In the implementation of FEM, the considered domain $\Omega$ is discretized into surface or body elements. The temperature inside a surface element $e$ can be obtained by following 
interpolation equation:

$$
u \approx \tilde{u}=\sum_{i=1}^{n_{e}} N_{i}\left(x_{1}, x_{2}\right) u_{i}
$$

where $n_{e}$ is the number of nodes for one element. $N_{i}\left(x_{1}, x_{2}\right)$ is the interpolation function. $u_{i}$ is the local temperature associated with the node with index $i$.

Substituting eqn (5) into the discrete functional formulation, invoking $\delta \Pi(u)=0$, we can obtain the following finite element equation for steady heat conduction problems

$$
\mathbf{K u}=\mathbf{R},
$$

where $\mathbf{K}$ is the stiffness matrix for heat transfer problems. Here, $\mathbf{K}$ is a symmetric matrix. Array $\mathbf{u}=\left[\begin{array}{llll}u_{1} & u_{2} & \cdots & u_{n}\end{array}\right]^{T}$ contains the nodal temperature. Vector $\mathbf{R}$ contains the temperature load. The elements $K_{i j}$ and $R_{i}$ of Matrix $\mathbf{K}$ and $\mathbf{R}$ can be expressed as:

$$
\begin{aligned}
K_{i j} & =\sum_{e} K_{i j}^{e}, \\
R_{i} & =\sum_{e} R_{q_{i}}^{e},
\end{aligned}
$$

where $K_{i j}^{e}$ and $R_{q_{i}}^{e}$ can be expressed as

$$
\begin{gathered}
K_{i j}^{e}=\int_{\Omega^{e}} k\left(\frac{\partial N_{i}}{\partial x_{1}} \frac{\partial N_{j}}{\partial x_{1}}+\frac{\partial N_{i}}{\partial x_{2}} \frac{\partial N_{j}}{\partial x_{2}}\right) d \Omega, \\
R_{q_{i}}^{e}=\int_{\Gamma_{2}^{e}} N_{i} t d \Gamma
\end{gathered}
$$

2.2 BE formulations for the heat transfer problems

For a $2 \mathrm{D}$ heat transfer problem, the corresponding boundary integral equation can be written as [21]

$$
c u(p)=\int_{\Gamma} u^{*}(Q, p) t(Q) d \Gamma(Q)-\int_{\Gamma} t^{*}(Q, p) u(Q) d \Gamma(Q),
$$

where $p$ represents the source point; $Q$ represents the field point; $c$ is a known value that depends on the geometric shape around the source point $p . u^{*}(Q, p)$ and $t^{*}(Q, p)$ denote the temperature and flux fundamental solution kernels, which are defined by

$$
\begin{aligned}
u^{*}(Q, p) & =-\frac{1}{2 \pi} \ln r, \\
t^{*}(Q, p) & =\frac{\partial u^{*}}{\partial r} \frac{\partial r}{\partial \mathbf{n}} .
\end{aligned}
$$

For the implementation of BEM, the boundary $\Gamma$ will be divided into $n$ elements $\Gamma_{\text {elem }}$ $($ elem $=1,2, \ldots, n)$. Then, the boundary integral eqn (11) can be written in a discretized form

$$
c\left(p_{i}\right) u\left(p_{i}\right)+\sum_{\text {elem }=1}^{n} \int_{\Gamma_{\text {elem }}} t^{*}\left(Q, p_{i}\right) u^{Q} d \Gamma(Q)=\sum_{\text {elem }=1}^{n} \int_{\Gamma_{\text {elem }}} u^{*}\left(Q, p_{i}\right) t^{Q} d \Gamma(Q),
$$


where

$$
u^{Q}=\sum_{j=1}^{n_{e}} N_{j} u^{j}
$$

and

$$
t^{Q}=\sum_{j=1}^{n_{e}} N_{j} t^{j}
$$

$\Gamma_{\text {elem }}$ is the boundary of element with index 'elem'; $p_{i}$ is the source point with index $i$.

By considering eqn (14) at a sufficient number of source points, we can get the system of equation

$$
\mathbf{H u}=\mathbf{G t},
$$

where $\mathbf{u}$ and $\mathbf{t}$ represent the vectors contained temperature and flow at boundary nodes. Matrix $\mathbf{H}$ is a square matrix containing a combination of the integrals of the $t^{*}$ kernel and coefficient $c$. $\mathbf{G}$ is a rectangular matrix of $u^{*}$ kernel integrals.

\section{COUPLING OF BEM AND FEM}

Considering the coupling of structure domain $\Omega^{F}$ and $\Omega^{B}\left(\Omega=\Omega^{F} \cup \Omega^{B}, \Omega^{F} \cap \Omega^{B}=\phi\right)$. In domain $\Omega^{F}$, the finite element method will be used. And boundary element method will be used in domain $\Omega^{B}$.

\subsection{Formulations of finite element method}

According to the nodal locations, eqn (7) can be written as

$$
\left[\begin{array}{ll}
\mathbf{K}_{o o} & \mathbf{K}_{o i} \\
\mathbf{K}_{i o} & \mathbf{K}_{i i}
\end{array}\right]\left\{\begin{array}{l}
\mathbf{t}_{F_{o}} \\
\mathbf{u}_{F_{i}}
\end{array}\right\}=\left\{\begin{array}{l}
\mathbf{R}_{F_{o}} \\
\mathbf{R}_{F_{i}}
\end{array}\right\}
$$

where $\mathbf{K}_{s u b}, \mathbf{t}_{s u b}, \mathbf{u}_{s u b}$, and $\mathbf{R}_{s u b}$ are the newly constructed matrices and vectors. $F_{i}$ represents the quantities related to the interface in the FEM domain. And $F_{o}$ indicates the quantities related to the non-interface part in the FEM domain.

\subsection{Formulations of boundary element method}

Similarly, based on the nodal locations, eqn (17) can be written as

$$
\left[\begin{array}{ll}
\mathbf{H}_{i i} & \mathbf{H}_{i o} \\
\mathbf{H}_{o i} & \mathbf{H}_{o o}
\end{array}\right]\left\{\begin{array}{l}
\mathbf{u}_{B_{i}} \\
\mathbf{u}_{B o}
\end{array}\right\}=\left[\begin{array}{ll}
\mathbf{G}_{i i} & \mathbf{G}_{i o} \\
\mathbf{G}_{o i} & \mathbf{G}_{o o}
\end{array}\right]\left\{\begin{array}{c}
\mathbf{t}_{B_{i}} \\
\mathbf{t}_{B o}
\end{array}\right\},
$$

where $\mathbf{H}_{\text {sub }}, \mathbf{G}_{\text {sub }}, \mathbf{u}_{\text {sub }}$ and $\mathbf{t}_{\text {sub }}$ are the newly constructed matrices and vectors. And the subscript $B_{i}$ represents the quantities related to the interface in the BEM domain. $B_{o}$ indicates the quantities related to the non-interface part in the BEM domain.

Along the interface between the domains $\Omega^{F}$ and $\Omega^{B}$, the continuity condition requires that temperature calculated for the $\Omega^{F}$ must equal the temperature calculated for the $\Omega^{B}$. And a similar relationship remains for the equilibrium condition along the interface, except that a negative sign must be given to account for the opposite directions of the outward boundary normal in the two domains. To use these relationships, eqn (19) can be transformed to the following form by setting $\mathbf{H}_{i o}=0$

$$
\left[\begin{array}{cc}
\mathbf{A}_{i i} & 0 \\
\mathbf{A}_{o i} & \mathbf{A}_{o o}
\end{array}\right]\left\{\begin{array}{l}
\mathbf{u}_{B_{i}} \\
\mathbf{u}_{B o}
\end{array}\right\}=\left[\begin{array}{ll}
\mathbf{B}_{i i} & \mathbf{B}_{i o} \\
\mathbf{B}_{o i} & \mathbf{B}_{o o}
\end{array}\right]\left\{\begin{array}{c}
\mathbf{t}_{B_{i}} \\
\mathbf{t}_{B o}
\end{array}\right\} .
$$


From eqn (20), we can obtain the following equation

$$
\mathbf{A}_{i i} \mathbf{u}_{B_{i}}=\mathbf{B}_{i i} \mathbf{t}_{B_{i}}+\mathbf{B}_{i o} \mathbf{t}_{B o}
$$

Then, eqn (21) can be expressed as

$$
\mathbf{t}_{B_{i}}=\mathbf{B}_{i i}^{-1} \mathbf{A}_{i i} \mathbf{u}_{B_{i}}-\mathbf{B}_{i i}^{-1} \mathbf{B}_{i o} \mathbf{t}_{B o} .
$$

The heat flux $\mathbf{t}_{B_{i}}$ in eqn (22), should be converted into the equivalent nodal flux $\mathbf{R}_{B_{i}}$ which is used in finite element method. And $\mathbf{R}_{B_{i}}$ can be expressed in the form

$$
\mathbf{R}_{B_{i}}=\mathbf{M}_{B} \mathbf{t}_{B_{i}}=\mathbf{K}_{B_{i}} \mathbf{u}_{B_{i}}-\overline{\mathbf{R}}_{B_{i}}
$$

where $\mathbf{M}_{B}$ is a transformation matrix and

$$
\overline{\mathbf{R}}_{B_{i}}=\mathbf{M}_{B} \mathbf{B}_{i i}^{-1} \mathbf{B}_{i o} \mathbf{t}_{B o}
$$

According to the continuity condition of interface, the coupling equation can be finally expressed as

$$
\left[\begin{array}{cc}
\mathbf{K}_{o o} & \mathbf{K}_{o i} \\
\mathbf{K}_{i o} & \mathbf{K}_{i i}+\mathbf{K}_{B_{i}}
\end{array}\right]\left\{\begin{array}{l}
\mathbf{u}_{F_{o}} \\
\mathbf{u}_{F_{i}}
\end{array}\right\}=\left\{\begin{array}{l}
\mathbf{R}_{F_{o}} \\
\overline{\mathbf{R}}_{B_{i}}
\end{array}\right\} .
$$

It should be noted that $\mathbf{K}_{B_{i}}$ obtained from BEM domain is asymmetric, which destroys the sparsity of the FE stiffness matrix. Therefore, we will replace $\mathbf{K}_{B_{i}}$ by an enhanced symmetric matrix $\mathbf{K}_{l}$. And the coupling equation in eqn (25) is converted as follows:

$$
\left[\begin{array}{cc}
\mathbf{K}_{o o} & \mathbf{K}_{o i} \\
\mathbf{K}_{i o} & \mathbf{K}_{i i}+\mathbf{K}_{l}
\end{array}\right]\left\{\begin{array}{l}
\mathbf{u}_{F_{o}} \\
\mathbf{u}_{F_{i}}
\end{array}\right\}=\left\{\begin{array}{l}
\mathbf{R}_{F_{o}} \\
\overline{\mathbf{R}}_{B_{i}}
\end{array}\right\}+\left\{\begin{array}{c}
0 \\
-\mathbf{K}_{B_{i}}+\mathbf{K}_{l}
\end{array}\right\}\left\{\mathbf{u}_{F_{i}}\right\},
$$

where

$$
\mathbf{K}_{l}=\frac{1}{2}\left(\mathbf{K}_{B_{i}}^{T}+\mathbf{K}_{B_{i}}\right) .
$$

\section{IMPLEMENTATION OF THE COUPLING WITH ABAQUS}

ABAQUS is a powerful engineering finite element software, including various types of element libraries and material model libraries. It is becoming an important tool for engineers to solve complex nonlinear problems. In this section, we will introduce the scheme that combines a self-written BEM-code and the commercial finite element software ABAQUS. The UEL (User Element Subroutine) [22] provided by ABAQUS will be used to realize the coupling of FEM and BEM. The implementation process is given as follows:

- As shown in Fig. 1(a), the considered domain is divided into two sub-regions, i.e. FE region and $\mathrm{BE}$ region. Then, the $\mathrm{FE}$ region needs to be divided into quadrilateral or triangular finite elements which can be easily solved by pre-process tools of ABAQUS. While, for the BE region, only boundary element is used (see Fig. 1(b)).

- In the coupling scheme, one single model including FE and BE parts (shown in Fig. 2(a)) should be built by the ABAQUS platform. Here the BE part is defined as a one-dimensional simplified FE part. It should be noted that the number of nodes at the interface between $\mathrm{FE}$ region and $\mathrm{BE}$ region must be equal. And the corresponding nodes will be connected by the command ' $t i e$ ', as shown in Fig. 2(b). Then the model can be analyzed as a pure FE model. 


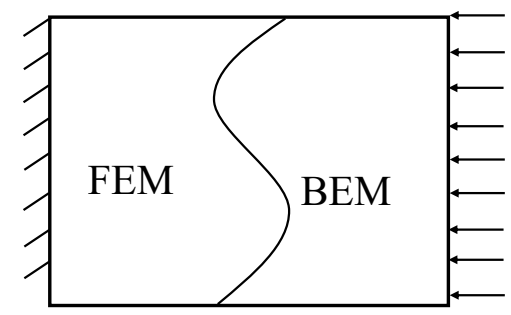

(a) A model divided into twon racrione

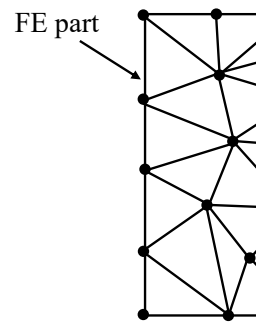

(a) FE p

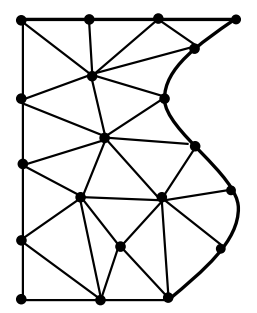

(h) Mach for the counlina model
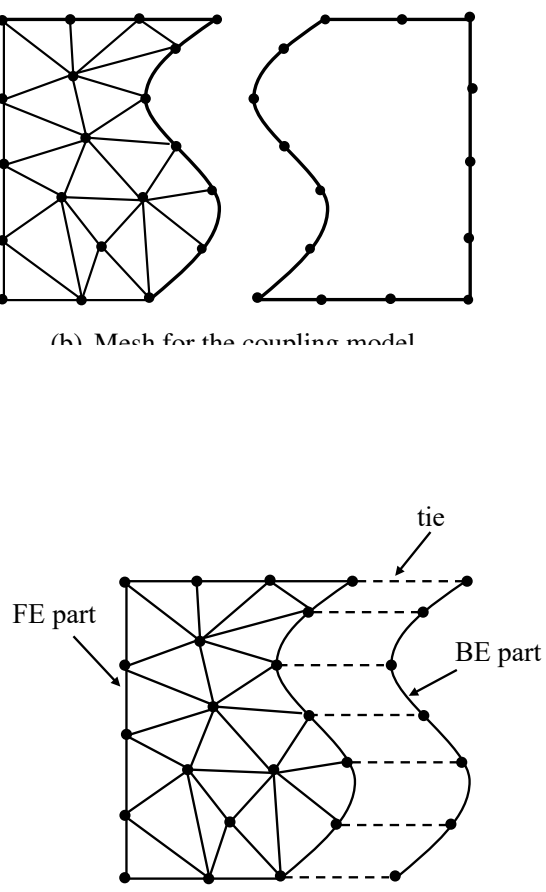

(b) Two parts connected by command ' $t i e$ '

Figure 2: Model built by ABAQUS.

\section{NUMERICAL EXAMPLE}

To demonstrate the accuracy and effectiveness of the new coupling approach, we examine a simple example about heat transfer problem. To carry out the accuracy and convergence analysis, a relative error defined in eqn (28)

$$
\text { Relative error }(\mathrm{RE})=\frac{\left|f_{\mathrm{num}}-f_{\mathrm{ref}}\right|}{\left|f_{\mathrm{ref}}\right|},
$$

wher $\epsilon$

ely.

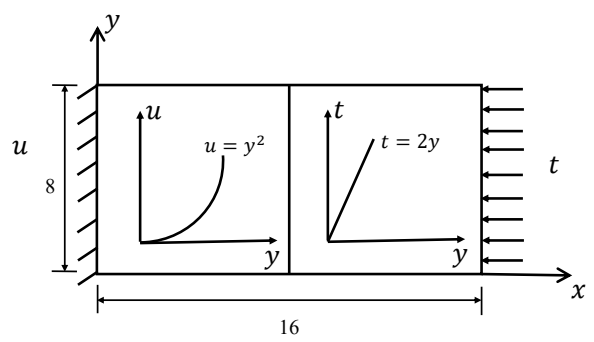

Figure 3: Considered rectangular plate.

As shown in Fig. 3, consider a $8 \times 16$ rectangular plate. The Dirichlet boundary condition is exerted on the left side of the plate and given by the $u=y^{2}$. The Neumann BC is exerted on 
the right side of the right region. Its given by the $t=2 y$. All the upper and lower boundaries are insulated, i.e. $\Delta u \cdot \mathbf{n}=0$. In the computation by the current coupling method, the plate in Fig. 3 is divided into two parts. And the left domain will be analyzed by FEM and the BEM will be used on the right part.

The mesh used in the coupling method is given in Fig. 4(a), from which we can see that the left region is divided into quadrilateral elements. And only the boundary is discretized in the right region. Fig. 4(b) shows the one-dimensional simplified FE part. And we can see from Fig. 4(b) that the number of nodes on the left side equals the number of nodes for the right curve.

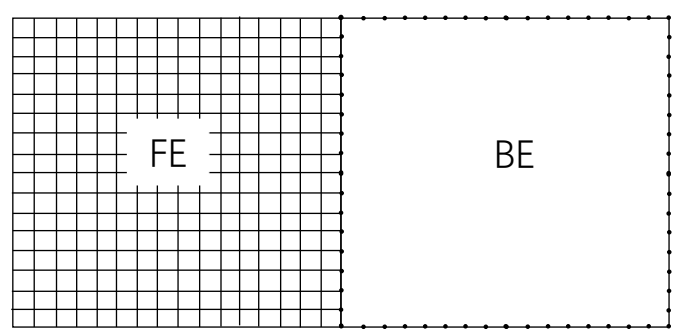

(a) Mesh for FE and BE parts

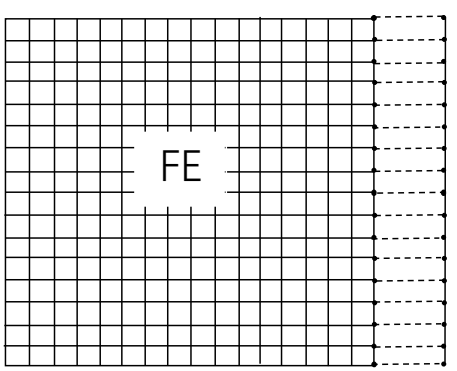

(b) Model established by ABAQUS

Figure 4: Mesh for the considered model.

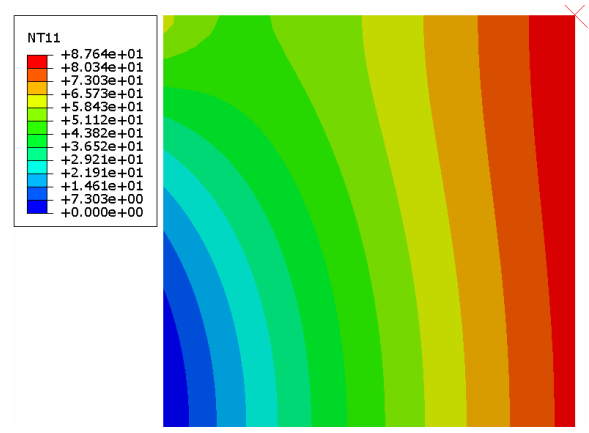

(a) Current coupling method

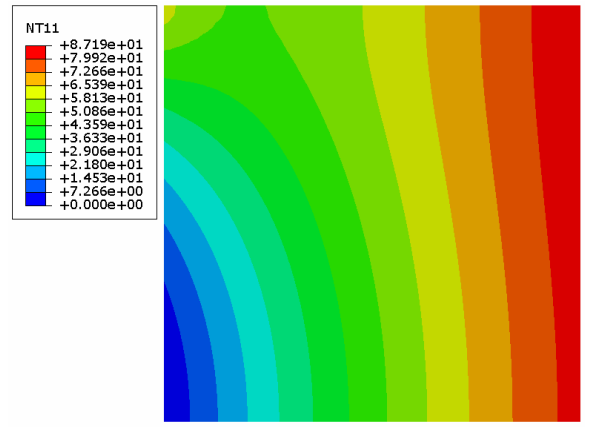

(b) FEM obtained by ABAQUS

Figure 5: Temperature distribution for different methods.

Here, a finite element model is constructed with ABAQUS to offer a reference solution. The element DC2D4 is used in this numerical example. The mesh consists of 1024 elements and 1089 nodes. The temperature distribution of the left region is given in Fig. 5(a) and 5(b) for the two methods. One can find that the results obtained by the current method are in good agreement with the FEM solutions.

To study the accuracy of the present method, some points are selected along the bottom boundary (with parametric equations $(x, 0), 0 \leq x \leq 8$ ) and the interface between the FEM part and BEM part. The temperature along different curves for different ndof are given in 
Fig. 6(a) and 6(b). Here, the number of degree of freedom (ndof) is the sum of ndof from FE part and BE part.

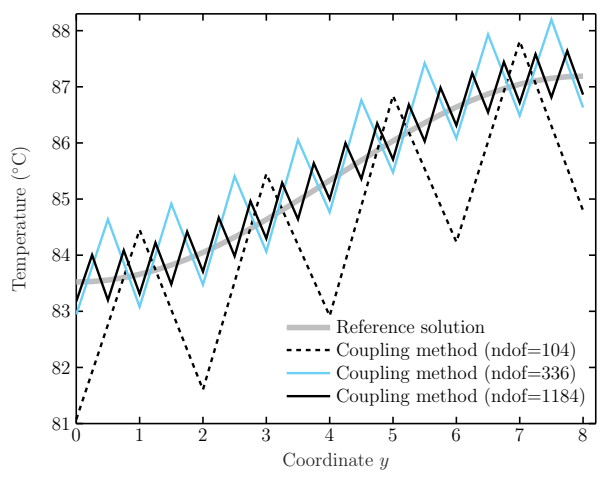

(a) Along the interface

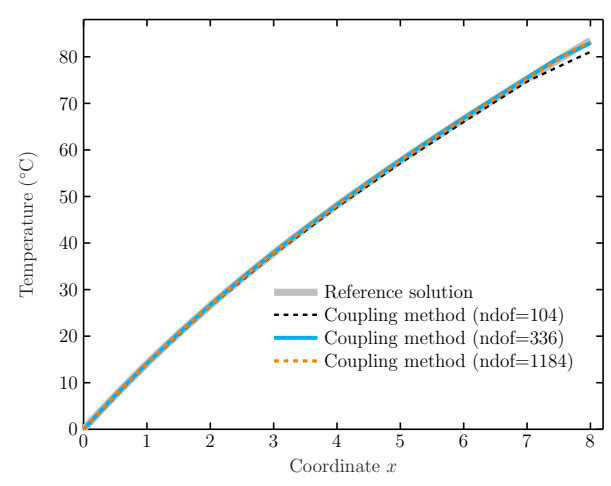

(b) Along the bottom boundary

Figure 6: The temperature calculated along different curves for different ndofs.

To study the convergence rate of the present method, a problem for which we can find the analytical solution is studied. The Dirichlet boundary condition on the left is changed as the fixed value 0 . And the Neumann boundary condition is changed to 1 . The exact temperatures for any point can be given as $u=x$. Fig. 7(a) and 7(b) show the relative errors of temperature as the ndof increases from 336 to 1184 . From Fig. 7(a) and 7(b), we draw the same conclusions as from Fig. 6(a) and 6(b), in that the relative errors of interfacial points are larger than that of boundary points. And the convergence of relative errors can be clearly seen for the two sets of points.

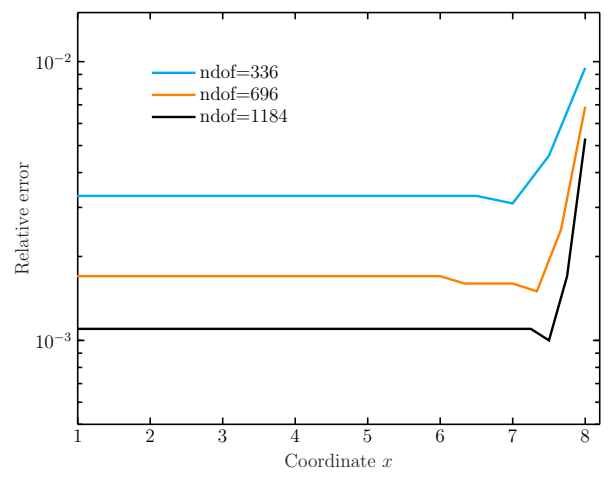

(a) Along the curve $(x, 0), 0 \leq x \leq 8$

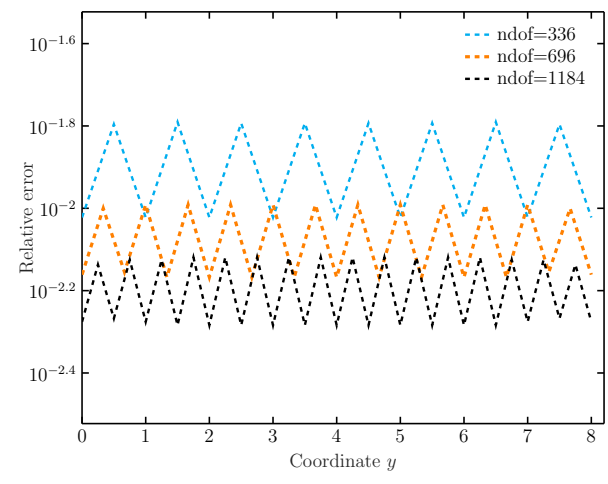

(b) Along the interface between BE and FE regions

Figure 7: Relative errors of temperature for different ndof.

\section{CONCLUSION}

Based on the finite element software ABAQUS, this paper presents a FEM-BEM coupling method and apply it to the steady-state heat transfer problem. In the implementation of 
coupling method, the UEL provided by ABAQUS is used to combine the self-written BEMcode and ABAQUS. The self-written BEM-code is used to obtain the effective stiffness matrix of the BE region. Then the resulted effective stiffness matrix and stiffness matrix of FEM region will form a coupling stiffness matrix, which will be solved by the solver of ABAQUS. Finally, a simple example about heat transfer problem is calculated by this method.

\section{ACKNOWLEDGEMENTS}

The research was supported by the National Natural Science Foundation of China (No. 12002009), the General Program of Science and Technology Development Project of Beijing Municipal Education Commission (No. KM202110005032), Beijing Postdoctoral Research Foundation and General Program of Science and Technology Development Project of BJUT.

\section{REFERENCES}

[1] Bailey, C., Thermal management technologies for electronic packaging: Current capabilities and future challenges for modelling tools. 2008 10th Electronics Packaging Technology Conference, IEEE, pp. 527-532, 2008.

[2] Ladeveze, P., Multiscale modelling and computational strategies for composites. International Journal for Numerical Methods in Engineering, 60(1), pp. 233-253, 2004.

[3] Blackburn, D.L., Temperature measurements of semiconductor devices - A review. Twentieth Annual IEEE Semiconductor Thermal Measurement and Management Symposium (IEEE Cat. No. 04CH37545), IEEE, pp. 70-80, 2004.

[4] Nardini, D. \& Brebbia, C., A new approach to free vibration analysis using boundary elements. Applied Mathematical Modelling, 7(3), pp. 157-162, 1983.

[5] Gu, Y., Chen, W. \& Zhang, C., Stress analysis for thin multilayered coating systems using a sinh transformed boundary element method. International Journal of Solids and Structures, 50(20-21), pp. 3460-3471, 2013.

[6] Gu, Y., He, X., Chen, W. \& Zhang, C., Analysis of three-dimensional anisotropic heat conduction problems on thin domains using an advanced boundary element method. Computers \& Mathematics with Applications, 75(1), pp. 33-44, 2018.

[7] Baynham, J., Adey, R., Murugaian, V. \& Williams, D., Simulating electro-coating of automotive body parts using BEM. WIT Transactions on Engineering Sciences, 54, 2007.

[8] Zhou, H.L., Han, H.S., Cheng, C.Z. \& Niu, Z.R., Inverse identification of heat boundary conditions for 2-D anisotropic coating structures. Applied Mechanics and Materials, Trans Tech Publications, 130, pp. 1825-1828, 2012.

[9] Zhang, Y.M., Gu, Y. \& Chen, J.T., Stress analysis for multilayered coating systems using semi-analytical BEM with geometric non-linearities. Computational Mechanics, 47(5), pp. 493-504, 2011.

[10] Zienkiewicz, O., Kelly, D. \& Bettess, P., The coupling of the finite element method and boundary solution procedures. International Journal for Numerical Methods in Engineering, 11(2), pp. 355-375, 1977.

[11] Von Estorff, O. \& Firuziaan, M., Coupled BEM/FEM approach for nonlinear soil/structure interaction. Engineering Analysis with Boundary Elements, 24(10), pp. 715-725, 2000.

[12] Elleithy, W.M., Al-Gahtani, H.J. \& El-Gebeily, M., Iterative coupling of BE and FE methods in elastostatics. Engineering Analysis with Boundary Elements, 25(8), pp. 685695, 2001. 
[13] Elleithy, W. \& Grzhibovskis, R., An adaptive domain decomposition coupled finite element-boundary element method for solving problems in elasto-plasticity. International Journal for Numerical Methods in Engineering, 79(8), pp. 1019-1040, 2009.

[14] Godinho, L. \& Soares Jr., D., Numerical simulation of soil-structure elastodynamic interaction using iterative-adaptive BEM-FEM coupled strategies. Engineering Analysis with Boundary Elements, 82, pp. 141-161, 2017.

[15] Liu, Z. \& Dong, C., Automatic coupling of ABAQUS and a boundary element code for dynamic elastoplastic problems. Engineering Analysis with Boundary Elements, 65, pp. 147-158, 2016.

[16] Soares Jr., D., FEM-BEM iterative coupling procedures to analyze interacting wave propagation models: Fluid-fluid, solid-solid and fluid-solid analyses. Coupled systems Mechanics, 1(1), p. 19, 2012.

[17] Pavlatos, G. \& Beskos, D., Dynamic elastoplastic analysis by BEM/FEM. Engineering Analysis with Boundary Elements, 14(1), pp. 51-63, 1994.

[18] Chien, C.C. \& Wu, T.Y., A particular integral BEM/time-discontinuous FEM methodology for solving 2-D elastodynamic problems. International Journal of Solids and Structures, 38(2), pp. 289-306, 2001.

[19] Boumaiza, D. \& Aour, B., On the efficiency of the iterative coupling FEM-BEM for solving the elasto-plastic problems. Engineering Structures, 72, pp. 12-25, 2014.

[20] Zienkiewicz, O.C., Taylor, R.L. \& Zhu, J.Z., The Finite Element Method: Its Basis and Fundamentals, Elsevier, 2005.

[21] Gong, Y., Trevelyan, J., Hattori, G. \& Dong, C., Hybrid nearly singular integration for three-dimensional isogeometric boundary element analysis of coatings and other thin structures. Computer Methods in Applied Mechanics and Engineering, 367, pp. 642673, 2019.

[22] Hibbitt, Karlsson \& Sorensen, ABAQUS/Standard: User's Manual, vol. 1, Hibbitt, Karlsson \& Sorensen, 1997. 